\title{
The dynamical nature of time
}

\author{
Antonio F. Rañada* $\quad$ A. Tiemblo ${ }^{\dagger}$
}

19 July 2011

\begin{abstract}
It is usually assumed that the " $t$ " parameter in the equations of dynamics can be identified with the indication of the pointer of a clock. Things are not so simple, however. In fact, since the equations of motion can be written in terms of $t$ but also of $t^{\prime}=f(t), f$ being any well behaved function, any one of those infinite parametric times $t^{\prime}$ is as good as the Newtonian one to study classical dynamics in Hamiltonian form. Here we show that, as a consequence of parametric invariance, the relation between the mathematical parametric time $t$ in the equations of dynamics and the physical dynamical time $\sigma$ that is measured with a particular clock (which is a dynamical system) is more complex and subtle than usually assumed. These two kinds of time, therefore, must be carefully distinguished. Furthermore, we show that not all the dynamical clock-times are necessarily equivalent and that the observational fingerprint of this non-equivalence has, curiously, the same form as that of the Pioneer anomaly, a still unexplained phenomenon.
\end{abstract}

\section{Introduction}

The main problem of dynamics is probably to understand in depth the role and meaning of the term "time". Two kinds of time are used in physics. On one side, the parametric time $t$, just an auxiliary mathematical element

\footnotetext{
*Facultad de Física, Universidad Complutense, 28040 Madrid, Spain. Email: afr@fis.ucm.es

†Instituto de Matemáticas y Física Fundamental, Consejo Superior de Investigaciones Científicas, Serrano 113b, 28006 Madrid, Spain. E-mail: Tiemblo@imaff.cfmac.csic.es
} 
which, strictly speaking, is not observable since any other time of the form $t^{\prime}=f(t), f$ being any well behaved function, serves equally to describe the motion of a dynamical system. On the other the time measured with particular clocks, say $\sigma$, which are dynamical systems obeying the laws of physics. The latter is a dynamical variable, for instance the angle of a pointer, and deserves therefore to be qualified as dynamical. The consequences of the existence of these two kinds of time, parametric and dynamical, have not been perhaps explored enough.

Here we show that the dynamical time $\sigma(t)$ measured by a clock $\sigma$, can be obtained as the solution of the equation of motion that characterizes the clock, of the form $\mathrm{d} \sigma / \mathrm{d} t=u(t)$, where $u(t)$ denotes here the "march" of the clock $\sigma$ with respect to the parametric time $t$. While $\sigma(t)$ has a real dynamical character, $t$ is just a mathematical parameter, which (i) has a purely auxiliary role to write the action and obtain the equations of motion, (ii) lacks any physical or dynamical nature, iii) it is a symbol that describes the evolutive character of the reality and (iv) is not observable. The differences between parametric and dynamical times could have significant consequences, since two dynamical clock-times, say $\sigma_{1}$ and $\sigma_{2}$, are not necessarily equivalent, so that there could be different times accelerating with respect to one another. The consequences of these arguments could be important; we just mention here two cases in which they could shed some light. First is the meaning of the cosmic time. Second, the fourth Heisenberg relation which requires that the time be a dynamical variable.

In order to write the equations of motion of a system in terms of really observable and dynamical quantities, what is done is to compare two motions, one of the system and the other of a standard clock. This requires the use of two principles. The first is the parametric invariance under transformations $t \rightarrow t^{\prime}=f(t)$, an important property of gravitation theories, the other is a principle of coherence, i.e., that the equations of motion of both the system and the clock be described by the same physical theory.

\section{Parametric invariance in classical dynamics}

Though the parametric time is a fundamental concept in classical dynamics, as said before, it has a non-dynamical character. As a consequence, there is no canonical momentum conjugate to $t$. Common wisdom assumes that this 
non-dynamical $t$ is measured with a clock, but this assumption must be submitted to a rigorous analysis. Note that it is always possible to synchronize two clocks at a certain initial time $t_{0}$, but what cannot be assured is that they will keep ticking at the same rate. This raises the question whether the equations of motion of dynamics depend or not on the march of the clocks, which implies the need to establish a parametric invariance principle. There exists a scheme in which all these problems can be solved by means of the introduction of the idea of a dynamical time [1, 2]. The $t$ variable appearing in this scheme is just a non-observable auxiliary parameter. In fact, the theory so constructed is parametric invariant, as happens also in general relativity.

In order to do that we replace the standard action $S=\int[p \dot{q}-H(p, q)] \mathrm{d} t$, by the alternative expression

$$
S=\int\left\{\Pi(t) \dot{\sigma}_{0}(t)+p(t) \dot{q}(t)-u(t)[\Pi(t)+H(p(t), q(t))]\right\} \mathrm{d} t,
$$

(overdot means derivation with respect to the auxiliary parameter $t$ ), where $\sigma_{0}(t), \Pi(t)$ are conjugate variables that describe the behavior of the clock, and $\Pi_{u}$, the momentum conjugate to $u(t)$, weakly vanishes.

The corresponding Hamiltonian is $\hat{H}=u[\Pi+H(p, q)]+\lambda \Pi_{u}$ where $\lambda$ is a Lagrange multiplier. The stability of the weak condition $\Pi_{u}=0$ implies the following first class constraint

$$
\Pi+H(p, q)=0,
$$

which induces the following reparametrization transformations $\delta \sigma_{0}=$ $\alpha(t), \delta q=\alpha(t) \dot{q}$ and $\delta p=\alpha(t) \dot{p}$ with $\alpha(t)$ being an arbitrary function.

The transformations induced by $\Pi_{u}$ allow then to interpret $u(t)$ as an arbitrary function, so that the Hamiltonian becomes

$$
H^{E}=u[\Pi+H(p, q)],
$$

Though this Hamiltonian reduces to a first class constraint, it contains a very realistic dynamical evolution, given by the Hamiltonian equations

$$
\dot{q}=u \frac{\partial H}{\partial p}, \quad \dot{p}=-u \frac{\partial H}{\partial q}, \quad u=\dot{\sigma}_{0}, \quad \dot{\Pi}=-\dot{H}=0 .
$$

It follows that

$$
\frac{\mathrm{d} q}{\mathrm{~d} \sigma_{0}}=\frac{\partial H}{\partial p}, \quad \frac{\mathrm{d} p}{\mathrm{~d} \sigma_{0}}=-\frac{\partial H}{\partial q}, \quad u=\frac{\mathrm{d} \sigma_{0}}{\mathrm{~d} t}, \quad \frac{\mathrm{d} H}{\mathrm{~d} \sigma_{0}}=0,
$$


equations that are full of dynamical significance. The first two are the canonical equations of motion with the dynamical time $\sigma_{0}$ as the time variable, in such a way that the evolution becomes a correlation between dynamical variables. The third one can be interpreted as the equation of motion (i.e., the "march") of $\sigma_{0}$ with respect to the parameter $t$. Notice that the total Hamiltonian $\hat{H}=u[\Pi+H(p, q)]+\lambda \Pi_{u}$ is the sum of two terms, describing, respectively, the physical system and the clock. The equation of motion of the second term, $H_{\text {clock }}=u \Pi+\lambda \Pi_{u}$, is precisely that of a clock $u=\mathrm{d} \sigma_{0} / \mathrm{d} t$.

Since this theory is invariant under reparametrization, we may fix, for instance, the gauge by the condition $\sigma_{0}=t$ (i.e., $u=1$ ), so that we recover the ordinary canonical formalism with $t$ being the Newtonian time. Notice that the choice of gauge means in fact to choose a clock.

The observations are performed with real clocks, which are dynamical systems, each one with a dynamical variable that is a well behaved increasing function of $t$ and can therefore be identified with a dynamical clock-time $\sigma(t)$, which can be used to fix the reparametrization gauge. As long as the observations make use of only the standard dynamical clock $\sigma_{0}$, the scheme is nothing else than the Hamiltonian equations. This may not occur, however, if a real clock $\sigma(t)$ with a different march is involved. In the latter case, the motion equations are (5), but with $\sigma$ and $\sigma_{0}$ instead of $\sigma_{0}$ and $t$, respectively,

$$
\frac{\mathrm{d} q}{\mathrm{~d} \sigma}=\frac{\partial H}{\partial p} ; \quad \frac{\mathrm{d} p}{\mathrm{~d} \sigma}=-\frac{\partial H}{\partial q} ; \quad u=\frac{\mathrm{d} \sigma}{\mathrm{d} \sigma_{0}},
$$

which describe the physics of a system in operationally realistic terms. This means that they do not refer to any unobservable parametric time but to $\sigma$, which is the time really observed by a real clock. The novelty is here the presence of the third equation (6), which is the dynamic equation of the second clock with respect to the standard one. The important fact for our purposes is that classical dynamics can be formulated as a parametrically invariant theory.

\section{The relativistic particle}

Before going into this section let us summarize the arguments of the previous one. Starting from a Hamiltonian theory with $n$ degrees of freedom, we introduced a new one (the dynamical time), in such a way that the motion 
equations become correlations between dynamical variables only (5). Nevertheless the new theory has a first class constraint (reparametrizations) that allows us to fix arbitrarily the value of $\sigma_{0}$. The only way to do that is to choose a new dynamical system, i.e., a real clock such as the Earth's motion or any other, with a well behaved dynamical variable $\sigma(t)$ as appears in (6). So in practice to fix the gauge of the symmetry under reparametrizations is to choose a clock. In other words, we measure a motion using another one as a standard. It must be underscored that, to completely formulate the equations of a dynamical system, the chosen clock must be specified.

The kinematics of the free particle in special relativity follows the same scheme. The parametric invariant action $\mathcal{S}=m c \int \mathrm{d} s$, corresponds to the Lagrangian (overdot means derivative with respect to an arbitrary time)

$$
\mathcal{L}=-m c \sqrt{\dot{x}_{0}^{2}-\dot{x}_{1}^{2}-\dot{x}_{2}^{2}-\dot{x}_{3}^{2}}
$$

It is easy to see that there is a first class primary constraint of the form

$$
p_{0}^{2}-p_{1}^{2}-p_{2}^{2}-p_{3}^{2}=m^{2} c^{2},
$$

that expresses the evident parametric invariance of the action. Due to the existence of this primary constraint not all the time derivatives of the coordinates can be obtained in terms of the momenta. Choosing now $\dot{x}_{0}$ as an arbitrary function of $t$, the Hamiltonian becomes

$$
H^{E}=\dot{x}_{0}\left(p_{0}+\sqrt{p_{1}^{2}+p_{2}^{2}+p_{3}^{2}+m^{2} c^{2}}\right),
$$

which reproduces (3) with $p_{0}$ playing the role of $\Pi$ and the square root being $H(p, q)$. It is clear thus that $x_{0}(t)$ must be interpreted as a dynamical time.

Let us take now the motion of a particle in a general metric tensor $g_{\alpha \beta}$, so that $\mathrm{d} s=\sqrt{g_{\alpha \beta} \mathrm{d} x^{\alpha} \mathrm{d} x^{\beta}}$. The Lagrangian is

$$
\mathcal{L}=-m c \sqrt{g_{\alpha \beta} \dot{x}^{\alpha} \dot{x}^{\beta}} .
$$

Note that, since the motion is geodesic, the components of the metric tensor are not dynamical variables but prescribed functions of the coordinates. Following the same procedure as in the previous case, the primary constraint is now $g^{\alpha \beta} p_{\alpha} p_{\beta}=m^{2} c^{2}$. Thus the Hamiltonian becomes

$$
H=\dot{x}^{0}\left[p_{0}-\left(N \sqrt{p_{i} p^{i}+m^{2} c^{2}}+p_{i} N^{i}\right)\right],
$$


where $i=1,2,3, N$ is the lapse, $N_{i}$ the shift and the Latin indices are raised and lowered with the three-dimensional metric. As we see, the situation is the same as in previous cases, $x^{0}$ playing the same role as the dynamical time.

It must be underlined that all the previous Hamiltonians are, in fact, first class constraints. They generate, however, well defined dynamical evolutions (see (5)-(6)). Notice that they contain two terms that describe i) the dynamical system which is studied and ii) a particular clock.

The case of the particle in a gravitational field $g_{\alpha \beta}$ illustrates the difference between the spatial coordinates $x^{i}$ and the temporal one $x^{0}$ since the former can be chosen arbitrarily while the latter needs an additional dynamical system (a real clock) in order for it to be fixed so that probably a $(3+1)$ spacetime is closer to the reality than a 4 -spacetime.

\section{The Einstein-Hilbert action}

General relativity was constructed to be a parametric invariant theory from its very foundation, as happens with any other diff-invariant theory. Its essential difference from the previous examples is that, in the former cases, the dynamical variables are the coordinates, defined in a non-dynamical metric. Conversely, in the latter, the dynamical variables are the components of the metric tensor, while the coordinates are auxiliary objects with no dynamical nature. Accordingly to our previous statements, we will take from now on a $(3+1)$-spacetime. In the ADM scheme [4] the Hamiltonian becomes

$$
H^{E}=\int \mathrm{d}^{3} x\left[N \mathcal{H}\left(q_{i j}, \pi^{i j}\right)+N_{i} \chi^{i}\left(q_{i j}, \pi^{i j}\right)\right]
$$

where $N$ and $N_{i}$ are the lapse and the shift, respectively, $q_{i j}$ the 3 -metric and $\pi^{i j}$ its canonically conjugate momentum. The absence of time derivatives of $N$ and $N_{i}$ determines the presence of primary first class constraints, which implies in turn that $N$ and $N_{i}$ are arbitrary functions. The secondary first class constraints $\mathcal{H}=0$ and $\chi_{i}=0$ fix the subspace in which the motion takes place. If one fixes $N_{i}=0$, the Hamiltonian becomes $H=\int \mathrm{d}^{3} x N \mathcal{H}$.

From this expression one could reproduce the same process followed before in the case of ordinary analytical dynamics. To interpret the dynamics described by a Hamiltonian such as (12) it suffices, maintaining $N_{i}=0$, to consider the meaning of $N$, defined as $\mathrm{d} \tau / \mathrm{d} t$ where $\mathrm{d} \tau$ is the proper time 
distance between two shells of the foliation. Note that $N$ is an arbitrary dynamical variable which plays thus the same role as $\dot{x}^{0}$ and $\dot{\sigma}$ in the previous cases: all of them are derivatives with respect to the parametric time. We find, therefore, that the dynamical time coincides with the proper time. Nevertheless, as is suitable to general relativity, the dynamical time is just a local time.

Fixing $N=1$ implies the use of proper time. In the case of the electromagnetism equations in a gravitational field, a geometrical contribution to the permittivity and permeability appear which modify the values of $\varepsilon_{0}$ and $\mu_{0}$ and thus the speed of light. This change is easily avoided by using the proper time as the dynamical time [3]. In any case, the speed of light is still a fundamental constant if measured with atomic clocks since the periods of atomic oscillations are obviously constant with respect to it. Curiously, atomic clocks measure proper time, notwithstanding the fact that they are quantum devices described by quantum physics, while the proper time is a classical concept.

The choice of a physical clock is then a most relevant question. The clock must comply with some obvious conditions. It must be a dynamical system, the solution of its equation of motion $\sigma(t)$ being a well behaved and monotonously increasing function of the parametric time $t$, as for instance the number of cycles of an harmonic oscillator or of the Earth rotation. Strictly speaking the fixing of a gauge is a mathematical question, though physically relevant since it is equivalent to the choice of a clock. It must be underscored that the complete description of a dynamical system needs to specify the clock which is used. This is a very important problem, specially for cosmological models.

It must be underscored that the previous arguments imply that the parametric invariance is the main characteristic of classical dynamics. I.e., this invariance states that the equations of motion are independent of the clock used to observe the trajectory. Otherwise said, it is a way to restrict to the time variable the principle of general covariance of relativistic physics.

Let us see what would happen if parametric invariance is not taken into account. For this purpose and in order to understand general relativity, simplified models have been proposed to obtain valuable information in areas such as quantum gravity or cosmology. The usual strategy is to kill some degrees of freedom. There is a way, however, to achieve the same result but going in the opposite direction, i.e., adding degrees of freedom. This is the case of the Husain-Kuchař model [5], which lacks the Hamiltonian (scalar 
constraint) in such a way that the number of degrees of freedom per space point grows from 2 to 3 . In such a theory, parametric invariance would be absent. The price to be paid then is that the four dimensional metrics that can be constructed seem to be degenerate. Without discussing this point here, it is important to state that the Husain-Kuchar model is a particular case of a more general theory (see [6] for details) that includes nondegenerate metrics if a dynamical time variable is present.

\section{A principle of coherence}

As was pointed out at the end of Section 1, when two clocks are involved the question of their coherence must be considered. There is no problem if the dynamics of both the system and the clock are governed by the same physical theory. This is because any discrepancy between two clocks must be solvable, from the theoretical point of view, in the frame of the theory itself. For instance, the effect of the tides on the Earth's rotation modifies the value of the day, an effect that can be calculated by taking into account the gravitation involved in the Earth-Moon system.

This requirement of coherence, which guarantees that the equation of motion of the clock (i.e., its march) is given by the same theory as that of the dynamical system, cannot be maintained when the clock and the system obey two different theories. This is the case when atomic clocks are used in classical general relativity. Lacking a quantum gravity theory, the equation of motion of the atomic clocks $\sigma_{2}(t)$ cannot be determined a priori and, consequently, it is not possible to compare it with the equation of motion $\sigma_{1}(t)$ of a classical clock. The only way to do so relies necessarily on empirical methods. Note that if it is found that the two marches are different, this does not necessarily imply a violation of parametric invariance.

The previous considerations certainly clarify the role of the clocks and the meaning of the word "time". The two main kinds of clocks used in physics are the astronomical and the atomic ones, which are dynamical systems based on classical and quantum physics, respectively. The solar system taken as a clock gives the ephemeris time while the vibrations of quantum systems measure the atomic one. Current wisdom assumes implicitly that these two types of clocks give the same time but, as explained before, this is not necessarily so. Indeed there is no a priori reason to postulate that two clocks beat at the same rate if they are based on two different theories, such as gravitation and 
quantum physics which are not only different but, even more, all efforts to unify them have failed up to now.

\section{Looking for observational evidence}

The arguments of this paper show that the difference between $t_{\text {astr }}$ and $t_{\text {atom }}$ is either nil or very small, otherwise an unexpected new effect should have been detected by now. Let us admit that it is non-nil. Because of the continuous improvement of measurement devices during the last decades, an observational test of the relative acceleration between these two times might already be available, although we could be unaware of this possibility. What's more, the effect could have been observed by now but without being properly interpreted. A provoking case could be a spaceship receding from the Sun. Since its trajectory is calculated with standard gravity theories that use astronomical time but it is measured with devices based on quantum physics that use atomic time, some anomaly could be observed. In fact the theory gives the ship's trajectory as a certain function parametrized by astronomical time $\mathbf{r}=\mathbf{r}\left(t_{\text {astr }}\right)$ but the observations see the same three-dimensional trajectory, although parameterized by atomic time and given by a different function $\mathbf{r}^{\prime}=\mathbf{r}^{\prime}\left(t_{\text {atom }}\right)$. The two times are related as $\mathbf{r}^{\prime}\left(t_{\text {atom }}\right)=\mathbf{r}\left(t_{\text {astr }}\right)$ (they are examples of the aforementioned clock-times $\sigma_{2}(t)$ and $\left.\sigma_{1}(t)\right)$. It is clear that they can be synchronized at a certain initial time so that $t_{\mathrm{astr}, 0}=t_{\mathrm{atom}, 0}=t_{0}$, but they will start to desynchronize progressively afterwards as

$$
\mathrm{d} t_{\text {atom }}=\left[1+a\left(t-t_{0}\right)\right] \mathrm{d} t_{\text {astr }}, \quad \text { with } \quad a=\frac{\mathrm{d}^{2} t_{\text {atom }}}{\mathrm{d} t_{\text {astr }}^{2}},
$$

where the small inverse time $a$ is the relative acceleration of $t_{\text {atom }}$ and $t_{\text {astr }}$, and $u=\mathrm{d} t_{\text {atom }} / \mathrm{d} t_{\text {astr }}=1+a\left(t-t_{0}\right)$ the march of $t_{\text {atom }}$ with respect to $t_{\text {astr }}$. Note that it is not necessary, at first order, to specify which one of the two times is $t$.

Defining the velocities of a mobile with respect to the two times as $v_{\text {atom }}=$ $\mathrm{d} \ell / \mathrm{d} t_{\text {atom }}$ and $v_{\text {astr }}=\mathrm{d} \ell / \mathrm{d} t_{\text {astr }}$, it follows that

$$
v_{\text {atom }}=\frac{v_{\text {astr }}}{u}, \quad \frac{\Delta v}{v}=-a\left(t-t_{0}\right),
$$

with $\Delta v=v_{\text {atom }}-v_{\text {astr }}$. As could have been expected, the observational fingerprint of the relative acceleration of the two clock-times would be a 
discrepancy between the expected and observed speeds of a mobile. This implies that the speed of light would depend on which clock-time is used: it is a fundamental constant only if measured with atomic clock-time. It must be so since the periods of the atomic oscillations are obviously constant with respect to $t_{\text {atom }}$, in fact they are its basic units (see [2, 10] where the details are explained). Note that (13)-(14) imply that if $a<0$, then $v_{\text {atom }}>v_{\text {astr }}$ while if $a>0$, then $v_{\text {atom }}<v_{\text {astr }}$ (assuming $t>t_{0}$ ). In the latter case, the ship would seem to lag behind the position predicted by gravitation theories.

In fact quite a similar lag has already been observed and has even a name: the Pioneer anomaly. Surprisingly, it remains unexplained more than thirty years after being discovered by Anderson et al. in 1980 [7, 8, 9] in spite of many efforts to account for it. What is important here is that the observational fingerprint of the anomaly has the same form as the second equation (14). What Anderson et al. found is that the frequencies of the two-way signals to and from the Pioneer 10 spaceship included an unexpected Doppler residual which did not correspond to any known motion of the ship. They were able to measure the value $a=(5.84 \pm 0.88) \times 10^{-18} \mathrm{~s}^{-1}$, although using the inverse time $a_{\mathrm{t}}=a / 2$, and suggested that $a_{\mathrm{t}}$ could be "like a nonhomogeneity of time" or a "clock acceleration" [7]. But they did not explain acceleration with respect to what, nor did they develop any theoretical analysis of this idea, assuming at first that $2 a_{\mathrm{t}}$ was just the measure of a real Doppler effect. However it was soon understood that this interpretation is neither compatible with the equivalence principle nor with the cartography of the solar system. For several years it was thought that systematics would be the most probable explanation of the anomaly (see the conclusions of [8]) but no error was found in spite of several different mathematical analyses of the data, including independent ones [11, 12, 13, 14, 15, 16]. Currently the so called thermal model is investigated but, up to now, it has not given a solution to the riddle [17, 18]. For a relation of the recent attempts to explain the anomaly, see [15], Section 2.3, [19], Section 2 or [9], Section 6. Up to now and more than thirty years after its discovery, the Pioneer anomaly remains without a generally accepted solution, even though it happens in our backyard, the solar system.

Note, however, that this work's arguments, based on the principle of parametric invariance, give a solution of the riddle. In fact, the authors of this paper proposed a model to explain this intriguing phenomenon [2, 10], in which the non-equivalence of $t_{\text {atom }}$ and $t_{\text {astr }}$ is due to the combination of the fourth Heisenberg relation and the unavoidable coupling between the quan- 
tum vacuum and the background gravitational potential $\Psi_{\mathrm{bg}}(t)$ that must pervade the universe. The acceleration of the clocks is given in that model as $a=\eta \dot{\Psi}_{\mathrm{bg}}(t)$, where $\eta$ is a pure number related to the electromagnetic properties of empty space and the overdot means time derivative. However, as the presence of $\Psi_{\mathrm{bg}}(t)$ indicates, that previous model is objectionable since the very idea of potential is not well defined in general relativity and cosmology, except in some cases as an approximation. On the other hand, the arguments used in this work do not use the concept of potential. They are based instead on the principle of parametric invariance which is a very fundamental principle in classical dynamics.

Nevertheless, the previous work [2, 10] has some interesting features. It can be applied to a limited region of space, using the potential of the nearby bodies, not the background one. Moreover it suggests a mechanism to explain the physical reasons of the time acceleration. In fact, it is clear that gravity surely affects the value of $t_{\mathrm{astr}}$, while the quantum vacuum does not, the opposite being true for $t_{\text {atom }}$. One example of the application of the previous model is given in reference [20], where it is shown that it is fully compatible with the cartography of the solar system.

\section{Conclusions}

1. Building on the principle of parametric invariance, it was shown that the concept of time is much more complex than is usually assumed. It is important, in particular, to distinguish between parametric time and dynamical time and to understand that two stable, accurate and good but different clocks can be non-equivalent. By this we mean that the times they measure could accelerate with respect to one another if they are based on different physical theories, as happens in the case of atomic and astronomical times,

$t_{\text {atom }}$ and $t_{\text {astr }}$, which are based on classical gravity and quantum electromagnetism, respectively. This could be stated by saying that the principle of parametric invariance has room for non-equivalent clock-times.

2. It is very important to understand that the description of a dynamical system can not be considered complete without the explicit mention of the chosen physical clock. This is specially true in cosmology problems.

3. Although these arguments might seem rather formal, they are also of practical importance. In particular, this work proposes an explanation of the Pioneer anomaly that is a refinement of a previous one and is fully 
compatible with the cartography of the solar system [2, 20]. It is based on the non-equivalence of the atomic time and the astronomical time, which happens to have the same observational fingerprint as the anomaly. The inverse time $a$ that characterizes the observations turns out to be the second derivative of $t_{\text {atom }}$ with respect to $t_{\text {astr }}$.

Acknowledgements We are indebted to Profs. A. I. G. de Castro, J. Martín and J. Usón for discussions.

\section{References}

[1] A. T. Hanson, T. Regge, and C. Teitelboim, Constrained Hamiltonian systems (Academia dei Lincei, Roma, 1976.)

[2] A. F. Rañada, and A. Tiemblo, Found. Phys. 38, 458-469 (2008).

[3] L. D. Landau, and E. M. Lifshitz, The Classical Theory of Fields, 4th revised English edn. (Pergamon Press, Oxford, 1975), section 90 .

[4] R. Arnowitt, S. Deser, and C. W. Misner, Phys. Rev. 116, 13221331 (1959).

[5] V. Husain, and K. Kuchař, Phys. Rev. D 42, 4070-4077 (1990).

[6] J. Barbero, A. Tiemblo, and R. Tresguerres, Phys. Rev. D 57, 61046112 (1998).

[7] J. D. Anderson, Ph. A. Laing, E. L. Lau, A. S. Liu, M. M. Nieto, and S. G. Turyshev, Phys. Rev. Lett. 81, 2858-2861 (1998).

[8] J. D. Anderson, Ph. A. Laing, E. L. Lau, A. S. Liu, M. M. Nieto, and S. G. Turyshev, Phys. Rev. D 65, 082004/1-50 (2002).

[9] S. G. Turyshev, and V. T. Toth, arXiv:1001.3686v2 19 Aug 2010; Living Rev. Relativity, 13, 4-169 (2010).

[10] A. F. Rañada, Found. Phys. 34, 1955-1971 (2004), and references therein.

[11] C. B. Markwardt, arXiv:gr-qc/0208046v1 16 August 2002. 
[12] A. Levy, B. Christoffe, P. Berio, G. Metris, J.-M. Courty, and S. Reynaud, Adv. Space Res. 43, 1538-1544 (2009).

[13] Ø. Olsen, Astron. Astrophys. 463, 393-397 (2007).

[14] V. T. Toth, Int. J. Mod. Phys. D 18, 717-741 (2009).

[15] S. G. Turyshev, V. T. Toth, L. R. Kellog, E. L. Lau, and K. J. Lee, Int. J. Mod. Phys. D 15, 1-56 (2006).

[16] V. T. Toth, and S. G. Turyshev, arXiv:0710.25561 14 Oct 2007.

[17] V. T. Toth, and S. G. Turyshev, arXiv:0901.4597v2 [physics.spaceph] 27 Feb 2009, and references therein.

[18] S. G. Turyshev, and V. T. Toth, Space Sci. Rev. 148, 149-167 (2010).

[19] S. G. Turyshev, M. M. Nieto, and J. D. Anderson, in: G. A. Mamon, F. Combes, C. Deffayet, and B. Fort (eds.), arXiv:gr/qc/0510081v1 17 Oct 2005, EAS Publications Series 20, 243-250 (2006).

[20] A. F. Rañada, and A. Tiemblo, arXiv:0909.0912 v1 [gr-qc] 4 Sep 2009. 